\title{
SPICE Compact Modeling of Bipolar/Unipolar Memristor Switching Governed by Electrical Thresholds
}

\author{
Fernando García-Redondo Student Member, IEEE, Robert P. Gowers, A. Crespo-Yepes, \\ Marisa López-Vallejo, Member, IEEE and Liudi Jiang
}

\begin{abstract}
In this work we propose a physical memristor/resistive switching device SPICE compact model, that is able to accurately fit both unipolar/bipolar devices settling to its current-voltage relationship. The proposed model is capable of reproducing essential device characteristics such as multilevel storage, temperature dependence, cycle/event handling and even the evolution of variability/parameter degradation with time. The developed compact model has been validated against two physical devices, fitting unipolar and bipolar switching. With no requirement of Verilog-A code, LTSpice and Spectre simulations reproduce distinctive phenomena such as the preforming state, voltage/cycle dependent random telegraph noise and device degradation.
\end{abstract}

Index Terms-Memristor, RRAM, ReRAM, Variability, RTN, Degradation, SPICE, Compact Model, Multi-level, Circuit Simulation, Temperature

\section{INTRODUCTION}

Resistive switching (memristor) technologies are a promising part of next-generation nonvolatile memory. Their low power consumption, high density, fast operation and great endurance, as well as the integration capabilities with standard CMOS circuitry put memristive technologies under the spotlight. However, resistive random access memory (ReRAM or RRAM) is only one of the several applications where memristor technology has promising applications. Memristor based reconfigurable hardware, material-implication logic design or neuromemristive systems -neuromorphic computing using memristors- provide a solid alternative for standard CMOS circuits.

Close attention has been given to the fabrication of smaller, faster and more reliable resistive switching devices, using both oxide and semiconductor materials. Furthermore, major efforts have been made to characterize and correctly model those devices' behavior, depending on the properties of the materials and their fabrication conditions.

Previous works [1], [2] have accurately studied and reviewed the electrical behavior of memristor devices, gathering

Fernando García-Redondo and Marisa López-Vallejo are with Technical University of Madrid - UPM, Spain (email: fgarcia@die.upm.es, marisa@die.upm.es). Robert Gowers and Liudi Jiang (r.gowers@warwick.ac.uk, 1.jiang@soton.ac.uk)are with University of Southampton, England. A. Crespo-Yepes (albert.crespo@uab.es) is with Universitat Autònoma de Barcelona, Spain.

This work was funded by the projects TOLERA TEC2012-31292, TOLERA2 TEC2015-65902 of the Spanish Ministry of Economy and Competitiveness, the Spanish NANOVAR Network and the Mobility Grant Program of the Consejo Social UPM, Spain. information of their internal dynamic processes for the design of a compact model. This is important because the creation of subcircuit compact components ready to be used by SPICElike circuit simulators is a key issue for resistive switching based architecture design, since the trade-off between accuracy and simulation length becomes a critical factor when a large number of cells are computed within crossbars or substantial neural networks.

Considering the memristor as a two terminal device, insight into a model description can be found through two different approaches. Physical compact models rely on the description of current-voltage relationships together with their dependence on a given set of internal variables (conductive filament geometry, dopant volume elongation, ion migration probability, tunneling distances, etc.) matching the characteristics of a specific physical device. Depending on the grade of complexity, physical approaches include a broad variety of models, from the simpler and more flexible ones that are more inaccurate [3]-[5], to the more accurate but complex ones able to model the physical behavior with high precision [6]-[10]. The compendiums [1], [2] describe in more detail examples of both accurate and inaccurate models. Consequently, physical models that match different devices generally present accuracy problems, while complex approaches, with a suitable fitting of the physical component behavior, can fit only a narrow set of devices. Additionally, as the associated computational load increases with the model complexity, the trade-off between speed and accuracy restricts the use of some of the models. Moreover, in some cases the high non-linearity of the model behavior requires extremely short simulation time steps, cluttering the transient computation, and occasionally leading to convergence problems related to hard-switching conditions [1].

On the other hand, phenomenological compact models focus on fitting the electrical magnitude relationships, and describing their evolution using mathematical variables which are not necessarily related to the physical variables. Phenomenological approaches obtain a broad flexibility regarding the range of described devices [1]. More recent solutions update and improve earlier versions such as [11]-[13], standing as a powerful solution for general modeling, and an effective application to neuromorphic computing [14].

In this work we present a novel solution based on the independent modeling of the device conduction mechanisms and device state. The proposed model fully relies on physical magnitudes and their inter-relations, defining the tuple electri- 
TABLE I

Model Comparison Against Best Well Known Models

\begin{tabular}{|c|c|c|c|c|c|c|c|}
\hline Model & $\begin{array}{c}\text { Physical or } \\
\text { Phenomenological }\end{array}$ & $\begin{array}{l}\text { Require } \\
\text { Verilog-A }\end{array}$ & $\begin{array}{c}\text { Bipolar or } \\
\text { Unipolar }\end{array}$ & $\begin{array}{l}\text { Variability \& } \\
\text { Degradation }\end{array}$ & State Magnitude & $\begin{array}{c}\text { Multilevel } \\
\text { Aware }\end{array}$ & $\begin{array}{c}\text { Pristine State } \\
\text { Aware }\end{array}$ \\
\hline $\begin{array}{c}\text { Proposed } \\
\text { Several Universities [7], [8], } 10] \\
\text { Yakopcic's [11], 114] } \\
\text { Biolek's and Dopant Drift } 13],[5],[6] \\
\text { TEAM's [13], [15] }\end{array}$ & $\begin{array}{l}\text { Physical } \\
\text { Physical } \\
\text { Phenomenological } \\
\text { Physical } \\
\text { Physical }\end{array}$ & $\begin{array}{l}\text { No } \\
\text { Yes } \\
\text { No } \\
\text { No } \\
\text { Yes }\end{array}$ & $\begin{array}{l}\text { Both } \\
\text { Bipolar } \\
\text { Both } \\
\text { Bipolar } \\
\text { Bipolar }\end{array}$ & $\begin{array}{l}\text { Static and dynamic } \\
\text { Static } \\
\text { Static } \\
\text { No } \\
\text { No }\end{array}$ & $\begin{array}{l}\text { Consumed Energy } \\
\text { CF elongation } \\
\text { Auxiliar } \\
\text { Dopant area length } \\
\text { Dopant area length }\end{array}$ & $\begin{array}{l}\text { Explicit } \\
\text { CF mplicit } \\
\text { Adaptable } \\
\text { No } \\
\text { Adaptable }\end{array}$ & $\begin{array}{l}\text { Yes } \\
\text { No } \\
\text { No } \\
\text { No } \\
\text { No }\end{array}$ \\
\hline
\end{tabular}

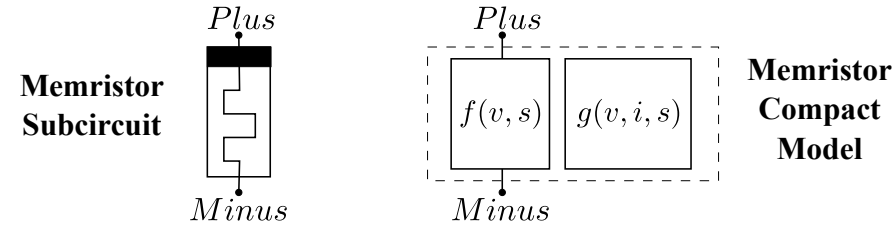

Fig. 1. Proposed Compact Modeling Design: bi-port component independently handling conduction module (left) and state module (right).

cal and resistive switching behavior.

While voltage and current thresholds are supported, the state modeling bases the device switching on electrical charge, flux or energy thresholds. It is applicable to both bipolar and unipolar devices, widening the range of devices that can be described, and therefore the proposed approach rivals in flexibility with the most suitable phenomenological approaches [11], [14]. In turn the conduction module accurately fits the conduction mechanisms of the physical component.

Fully compatible with SPICE simulators, the presented model does not require implementation in Verilog-A or CMI to accurately match physical components. Although components written in Verilog-A or CMI allow more complex schemes to be emulated, they have a major drawback: widely used SPICE simulators do not allow Verilog-A or CMI code execution, restricting its simulation on several platforms.

The main contributions of the proposed model are as follows:

- Accurate modeling of dynamic resistance cleanly mimicking physical device response.

- Effective switching behavior for bipolar/unipolar devices.

- Availability of cycle and switching event count information for all time, becoming a resource for additional characteristics such as variability.

- Variability awareness.

- Provision of variability dynamics and resistive state retention handling, defining how the device degrades through time/cycle stress.

- Explicit support for multi-level storage. Unlike CF based models [7], [8], [10], the explicit definition of multiple states allows more detailed level descriptions. This also allows the modeling of Pristine State -the initial High Resistive State (HRS) prior any electroforming-

- The full source code for those bipolar and unipolar devices, together with additional materials can be found at http://vlsi.die.upm.es/memristor_spice_model

Table II summarizes the key characteristics of the proposed model comparing it against the best well known compact models presented in literature.

The paper is structured as follows. First, we present the proposed memristor model. Section III describes the variability handling methods included within the modeling. The simulation results fitting two different physical devices are shown in Section IV. Some concluding remarks can be found in Section V. Finally, Appendix A includes LTSPICE source code describing a multilevel bipolar memristor.

\section{PROposed MODEL}

The proposed model is composed of two different subcomponents which allow matching to the device behavior (Figure 11. The Conduction Module, addressing the memristor component interface through the ports Plus and Minus, models the dynamic resistance; in turn, the State Module handles the device state.

The component behavior is defined by the transient signal set, composed of the input voltage $v(t)$, the current flowing through the device $i(t)$, and the device state vector $s(t)$. This vector contains all the information related to cycle count, switching thresholds, energy and resistance state. In this paper we will simplify the notation of the electrical and state equations, and their dependence on time will become implicit: consequently, as an example, $v(t)$ will be denoted as $v$.

The equations describing the signal relation of the different submodules can be written using the generalized system

$$
\begin{aligned}
i & =f(v, s) \\
\dot{s} & =g(v, i, s) .
\end{aligned}
$$

This equation system matches most devices' behavior [1], and thereby both physical and phenomenological models specify $f$ and $g$ functions to define how the model runs.

\section{A. Conduction Module Modeling}

The Conduction Module is responsible for the computation of the current which flows through the device. This depends on the voltage and the memristor state. Denoting the first component of the state vector $s^{(1)}$ as the discrete value referring the device level, within our proposed model the current function

$$
i=f(v, s)
$$

takes the form, for different $N$ conduction levels,

$$
i= \begin{cases}f_{1}(v, s) & \text { if } s^{(1)}=s_{1} \\ f_{2}(v, s) & \text { if } s^{(1)}=s_{2} \\ \cdots & \\ f_{N}(v, s) & \text { if } s^{(1)}=s_{N} .\end{cases}
$$




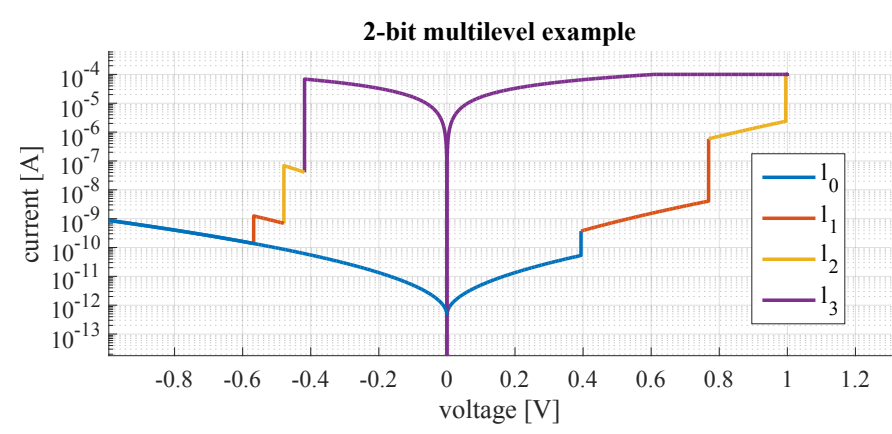

Fig. 2. Example of a simulated 2 bit multilevel device, with three different Schottky $\left(l_{0}, l_{1}\right.$ and $\left.l_{2}\right)$ and one ohmic conduction schemes $\left(l_{3}\right)$.

This approach, completely covering the device state design space, allows multilevel device modeling such as [16]. Figure 2 shows an example of a simulated 2 bit multilevel memristor using the proposed scheme. The bipolar device experiences three partial SET events followed by their corresponding partial RESETs. Compared against different approaches with no explicit multi-level definition (Table \), the proposed model eases tuning of each level's conduction properties, including degradation and variability. As an example, the Schottky characteristics exhibited in Figure 2 were captured with no effort.

For a device with two-resistance levels and no pristine state description, equation (3) gets simplified to the basic system

$$
i= \begin{cases}f_{o n}(v, s) & \text { if } s^{(1)}=s_{o n} \\ f_{o f f}(v, s) & \text { if } s^{(1)}=s_{o f f}\end{cases}
$$

where $f_{o n}$ and $f_{\text {off }}$ are the low and high resistance state currents, respectively.

The state currents $f_{j}$ are modeled using different methods depending on the specific device. As an example, low resistance state dependence on voltage is usually simplified to a single resistance with temperature dependence in both oxidebased [7], [8], [17] and semiconductor based devices [18]. However, the model accepts different schemes to be used, such as an in-series diode-resistance approach, improving the simulation accuracy [19].

Even though some works model the involved conduction mechanisms of the higher resistance states using dioderesistance schemes [9], in most cases the voltage-current relation is described by basic conduction processes like the ones described in Table [II Modeling such processes using behavioral current/voltage sources allows us to accurately mimic the device behavior. Some devices require complex schemes where not only individual conduction process take place but concurrent processes occur [17]. Our model fully covers this requirement allowing multi-contribution schemes, where distinct current sources contribute to define the global one.

In case the device conduction process is not defined by an analytical function, or further measurement data is required, we could model the varying resistance of the device as a behavioral resistor. The behavioral resistor value is determined by an auxiliary function defined using the circuit simulator user defined functions syntax [25]. Taking advantage of the
TABLE II

EXAMPles of CONDUCTION PROCESSES COMPATIBLE With SPICE-LIKE BEHAVIORAL SOURCES

\begin{tabular}{|c|c|}
\hline Process & Expression \\
\hline Trap Assisted [20] & $J \propto a_{1}\left(\frac{V}{d}\right)^{a_{2}}$ \\
\hline $\begin{array}{ll}\text { Generalized } & \text { Tunneling } \\
\text { Temperature } & \text { Dependent } \\
\text { (II) [7], [8] } & \end{array}$ & $J \propto \exp \left(\frac{-g}{g_{0}}\right) \sinh \left(\frac{V}{k T V_{1}}\right)$ \\
\hline Tunneling [6] & $J \propto \mathscr{C}^{2} \exp \left[-\frac{4 \sqrt{2 m^{*}}\left(q \phi_{B}\right)^{3 / 2}}{3 q \hbar \mathscr{C}}\right]$ \\
\hline $\begin{array}{l}\text { Schottky Thermionic } \\
{[17],[18],[21]}\end{array}$ & $J=A^{* *} T^{2} \exp \left[-\frac{\left({ }^{{ }_{D}} \quad \sqrt{ } 4 \pi \epsilon\right)}{k T}\right.$ \\
\hline Poole-Frenkel [22], [23] & $J \propto \mathscr{C}\left[-\frac{{ }^{T}\left(\pi_{B}\left(\frac{V}{\pi}\right)\right.}{k T}\right.$ \\
\hline Ohmic [17, [18], 24] & $J \propto \mathscr{C} \exp \left[-\frac{\Delta E_{a c}}{k T}\right]$ \\
\hline $\begin{array}{l}\text { Space-charge-limited } \\
\text { 24] }\end{array}$ & $J \propto \frac{9 \epsilon \mu V^{2}}{8 d^{3}}$ \\
\hline QPC [2] & $I=\frac{2 e}{h}\left\{e V+\frac{l n}{\alpha}\right.$ \\
\hline
\end{tabular}

$V$ and $d$ are the voltage applied between the electrodes and the insulator thickness respectively.

$\mathscr{C}$ refers to the electric field, $A^{* *}$ stands for the effective Richardson constant. $g, g_{0}, V_{0}, V_{1}$ is the gap distance and matching parameters.

$\phi$ is the materials barrier height, while $\epsilon$ is the insulator permitivity. $\Delta E_{a c}$ is the activation energy of electrons.

$\alpha$ and $\beta$ are parameters related to barrier thickness and fraction of dropped voltage respectively.

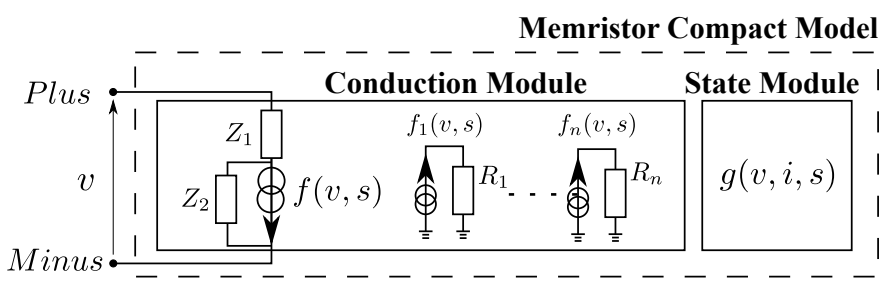

Fig. 3. N-state conduction module scheme using behavioral sources.

SPICE simulator capabilities, the behavioral resistor may refer a piecewise $(P W L)$ function defining the voltage and state dependent resistance based on previous voltage-current measurements that build up the pairs $v_{i}-r_{i}$.

Summarizing, our proposed model accepts individual/cumulative conduction schemes with which it models the current flow at multilevel states for different devices/technologies. The general subcircuit of a memristor device with $N$ different resistive levels modeled using $N$ independent current behavioral sources is shown in Figure 3 This approach allows us to include additional helpful effects such as the compliance current or control sentences shown in [1]. An example of a constraint current source modeling an $N$ state device using the approach described in Figure 3 would be represented by 


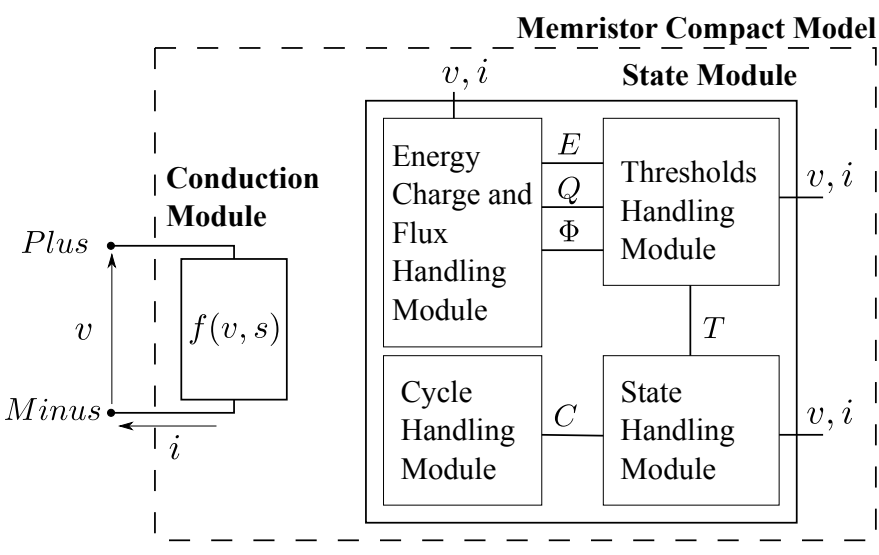

Fig. 4. Proposed Device State Handler Module.

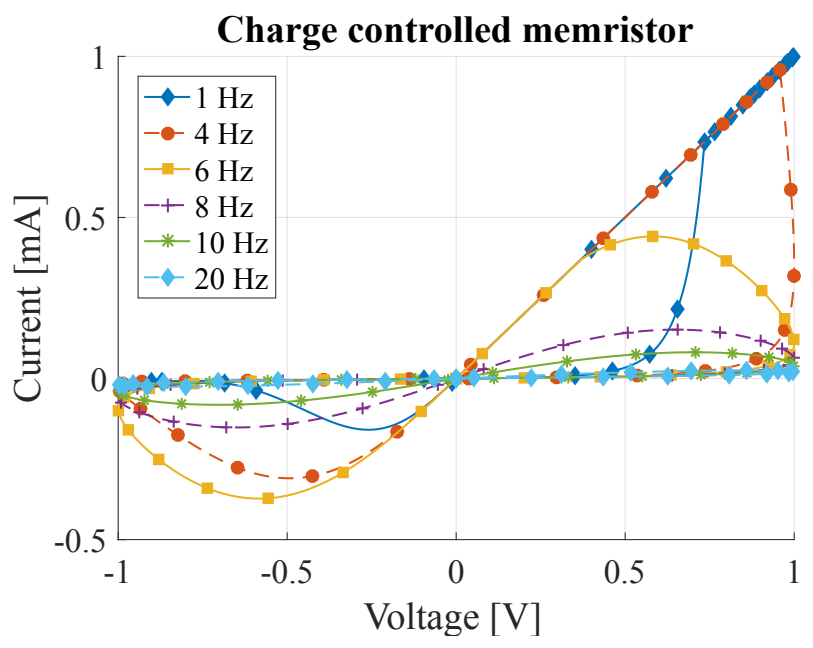

Fig. 5. Characteristic hysteresis loops of a charge controlled memristor fed with different sine voltages. The compact model was built following the scheme shown in Figure 4 using a charge handling module to control the device state.

the following equation:

$$
i(v, s)=\max \left(-c I, \min \left(c I, f_{j}(v, s)\right)\right) .
$$

Here, the current through the device is limited by the compliance current parameter $c I$.

In the same manner, the scheme shown in Figure 3 allows parasitic-overshoot effects [7], [17], [26] modeling. To that end, parasitic impedances $Z_{1}$ and $Z_{2}$ are attached in series and parallel to the global current source.

\section{B. State Module Modeling}

Several works propose models whose behavior depends on voltage or current thresholds that trigger different mechanisms unleashing a state change in the device [1], [11], [13].

In this work we propose an extension of this concept, broadening the threshold mechanisms that define the switching. Figure 4 describes the components the State Handler Module is composed of. Basically, the device state will depend on the previous state and the device electrical inputs.

This scheme is compatible with the simple approach of voltage/current boundary threshold, leaving the magnitudes energy, charge and flux out of the threshold management and using instant voltages and currents to trigger the switching mechanisms.

Moreover, the model is enriched, allowing energy, charge or flux to be used on the simulated device as the triggering magnitude that releases the switching events. Following the idea shown in [27]-[31], this model computes the energy, charge or flux levels at which the device experiences a state change. Using individual submodules, we calculate the energy, charge or flux applied to the device in order to define the switching, the cycle and event count, building up the whole state vector $s$. Following this approach Figure 5 shows the characteristic pinched hysteresis loop for a charge controlled device similar to [3].

Compared to Verilog-A implementations [7], [8], where different variables can be used, in SPICE-like subcircuit design we need to use signals to store the related information. Therefore, the energy, charge, flux or cycle/event count computations require individual signals, commonly node voltages, to define their value.

Instead of using capacitors to integrate magnitudes [4], the energy computation can be accelerated [1] using the time integral function provided by the SPICE-like simulator idt. On the other hand a user defined function, switching, performs the switching conditions verification. Taking energy as the magnitude managing the switching, switching would be described by equation 6 .

$$
s_{\text {next }}^{(1)}=\operatorname{switching}\left(E, P, s_{\text {current }}^{(1)}\right)
$$

There we name $P$ as the matrix whose elements $p_{j k}$ define the energy thresholds to switch from state $j$ to state $k$. This state-controlling scheme was used for the multilevel simulation show in Figure 2. Appendix A includes the SPICE code associated with switching function.

Pristine State Modeling: Using the proposed conduction and switching schemes allows us to model the pristine (conductive filament preforming) state. The ability of including this additional state enriches the model capabilities. By defining the conduction mechanism and forming energy $-e_{p^{-}}$, the pristine state can be easily included in the netlist:

$$
s_{\text {next }}^{(1)}=\left\{\begin{array}{lc}
s_{\text {pristine }}^{(1)} & \text { if } E<e_{p} \\
\text { switching }\left(E, P, s_{\text {current }}^{(1)}\right) & \text { otherwise. }
\end{array}\right.
$$

Cycle and Event Count Computation: As explained in Section III the ability to compute how many switching events a device has suffered is essential to accurately model its degradation. Using the magnitude that defines the switching -energy, charge or flux- we propose a novel scheme that allows the device cycling count to be stored. For the sake of simplicity, equations 8 and 9 will suppose devices with two states and an initial HRS. Let $E$ be the computed energy, parameters $p \_s e t$ and $p \_r e s e t$ the required energy levels to perform the SET and RESET respectively, and $p \_c y c l e=p \_s e t+p \_r e s e t$. For 


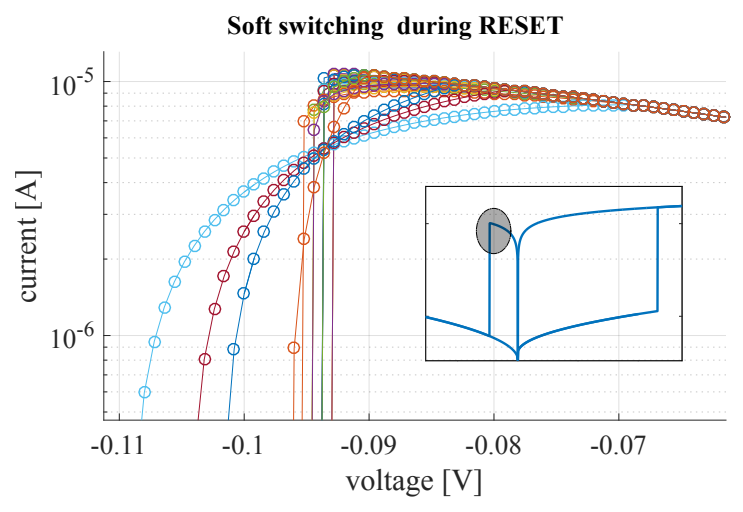

Fig. 6. Proposed soft-switching mechanism: the softening functions make use of the auxiliary variable $b$ to soften the state switching. Different thresholds and $p$ values produce the shown effects during reset process.

unipolar devices we can compute the state vector $s$ as

$$
\begin{aligned}
E & =\int_{0}^{t} v \times i d t \\
\text { cycles } & =\left\lfloor\frac{E}{p \_c y c l e}\right\rfloor \\
\text { extra_set } & = \begin{cases}1 & \text { if }\left(E-\text { cycles } \times p \_c y c l e\right) \\
0 & \text { otherwise }\end{cases} \\
\text { events } & =2 \times \text { cycles }+ \text { extra_set }
\end{aligned}
$$

The same methodology can be applied to bipolar devices. However, positive contributions $E_{+}$are required to be treated independently from negative contributions $E_{-}$:

$$
\begin{aligned}
\text { cycles } & =\left\lfloor\frac{E_{-}}{p_{-} \text {reset }}\right\rfloor \\
\text { events } & =\left\lfloor\frac{E_{-}}{p_{-} \text {reset }}\right\rfloor+\left\lfloor\frac{E_{+}}{p_{-} \text {set }}\right\rfloor
\end{aligned}
$$

Soft Switching Events: We can avoid hard-switching events by introducing an additional variable $b$ that softens the transition between states. For a device experiencing a transition from state $s_{j}^{(1)}$ to state $s_{j+1}^{(1)}$, where $i_{j}$ and $i_{j+1}$ are their corresponding current levels, the current switching can be softened if the global current is redefined as

$$
i=\ldots+w_{j}(b) \times i_{j}+w_{j}(1-b) \times i_{j+1}+\ldots
$$

where $w_{j}(b)$ are the softening functions that control the different state's current contributions. The softening variable $b$ can be described based on the corresponding energy thresholds

$$
\begin{aligned}
a 1 & =\left\{\frac{E_{j}}{e_{a 1}}\right\} \\
a 2 & =\left\{\frac{E_{j}}{e_{a 2}}\right\} \\
b & = \begin{cases}\min (1, \max (0, a 1)) & \text { if } s^{(1)}=s_{j} \\
1-\min (1, \max (0, a 2)) & \text { if } s^{(1)}=s_{j+1}\end{cases}
\end{aligned}
$$

where $E_{j}$ is the total/partial energy (depending on the device type), and $e_{a 1}$ and $e_{a 2}$ the energy thresholds required to perform the transitions $s_{j}^{(1)} \rightarrow s_{j+1}^{(1)}$ and $s_{j+1}^{(1)} \rightarrow s_{j}^{(1)}$ respectively.
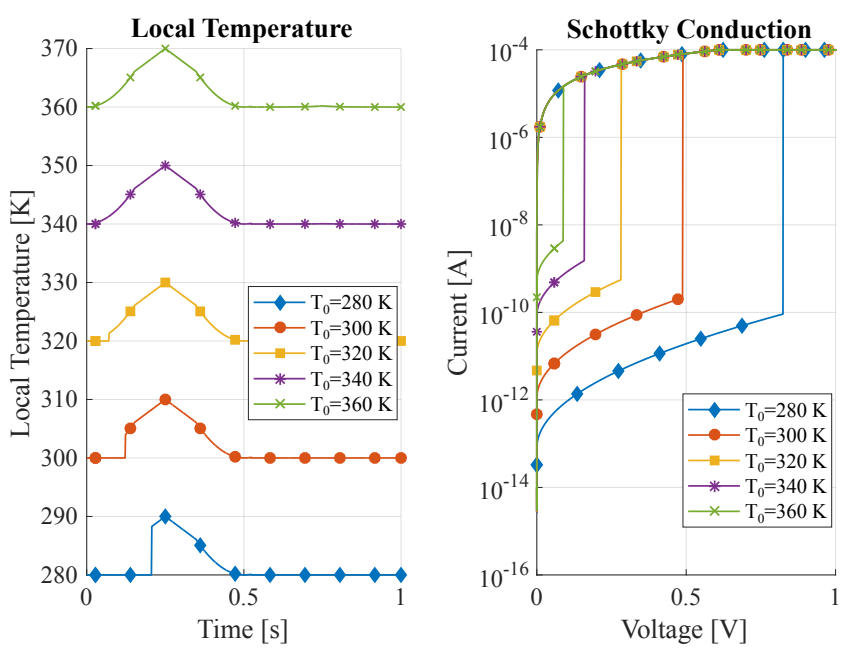

Fig. 7. Device's local temperature during the SET process and computed voltage-current relation, varying circuit temperature $T_{0}$.

The softening functions $w_{j}(b)$ make use of the state variable $b$ to control the partial contributions of $i_{j}$ and $i_{j+1}$. The model designer is able to customize these functions to better adapt the switching mechanism to the physical measures. Figure 6 describes one of the possible softening schemes for bi-state devices, together with the produced effects. In these proposed functions the partial contributions depend on two thresholds, b_th_set and b_th_reset, and the parameter $p$, which manages the softening in the following expressions:

$$
\begin{aligned}
f_{\text {set }}(b) & = \begin{cases}1 & \text { if } b \geq b_{t h \_s e t} \\
\left(\frac{b}{b_{t h_{-} s e t}}\right)^{p} & \text { if } b<b_{t h_{-} s e t}\end{cases} \\
f_{\text {reset }}(b) & = \begin{cases}1 & \text { if } b \leq b_{\text {th_reset }} \\
\left(1-\frac{b-b_{t h \_ \text {reset }}}{1-b_{\text {th_reset }}}\right)^{p} & \text { if } b>b_{\text {th_reset }}\end{cases}
\end{aligned}
$$

\section{Extensibility and Verilog-A Implementation}

The modular design of the proposed scheme allows its extension in order to consider additional phenomena. As an example, temperature dependence has proven a key factor in the device behavior [7], [17], [32]. Each of these extensions can be considered as independent behavioral sources for their later reference in dependent signals. The cited temperature influence can be directly incorporated within our model in both conduction mechanisms and energy thresholds computation by simply altering the behavioral source's nominal expressions. Figure 7 presents the results after the incorporation of local thermal behavior due to Joule-heating [7], [8].

Once the conduction mechanisms and the threshold parameters are defined, the present model can be easily ported to the Verilog-A language, with the consequent speed up of the circuit simulation. Each behavioral source is directly translated into an independent variable, following the same approach shown in [7]. Additionally, Verilog-A is more flexible regarding the use of user defined functions or variable data types (such as arrays), which eases the implementation of the methods adopted to reproduce variability effects shown in the next section. 
However, even though some circuit simulators such as Cadence spectre allow Verilog-A code execution, widely used SPICE simulators like LTSP ICE do not allow Verilog-A co-simulation, accepting SPICE-like code only. Consequently, research community may benefit from the SPICE proposed compact model.

\section{Variability \& State Retention Modeling}

Variability in memristors is one of the most concerning issues to be solved in memristive applications. Its effects are so visible that some works even use RRAMs to provide random pattern based circuit modules [33]. Three distinct kinds of variability are expected to occur in resistive switching devices [34]:

- Inter-Device Variability. Device to device variations in size, thickness, ion-concentrations, etc.

- Intra-Device Variability. Small cycle-to-cycle fluctuations caused by the stochastic nature of generation and recombination of oxygen vacancies and ion migration.

- Read Current Fluctuations, called Random telegraph noise $(R T N)$, is a variation in the measured current under constant bias during reading operations. It can be caused either by the electron capture and emission processes that inherently exist in oxides with high defect concentrations, or by atomic changes in the conducting filament.

Therefore, several works have studied the variability in different devices [34]-[37] and its inclusion into some Verilog-A models such as the one presented in [7].

Our proposal, fully compatible with SPICE-like simulators, allows modeling variability not only by using probability density functions but also by the direct injection of random patterns extracted from physical measurements. Figure 8 shows the scatters and histograms after measuring and normalizing the required energy to perform consecutive cycles (SET and RESET process). Therefore we can extract from those graphs the energy threshold mean and standard deviation values. By contrast, in cases where the random data does not follow a suitable function, measurements directly from the scatter are used.

The energy thresholds, the current flowing through the device or any other signal requiring variability injection can be modeled as sources whose value is composed of nominal and variable contributions. The variable contribution is described through a user defined function, $r_{j}()$, that can directly refer to the probability density function [35], [36]

$$
i= \begin{cases}r_{1}(v, s) f_{1}(v, s) & \text { if } s^{(1)}=s_{1} \\ \cdots & \\ r_{N}(v, s) f_{N}(v, s) & \text { if } s^{(1)}=s_{N}\end{cases}
$$

The probability density function can be generated using the uniform random generator function built into the circuit simulator. If the circuit simulator does not support this feature, we can extract the random pattern by adding an additional zero amplitude voltage source affected by uniform noise.

On the other hand, by using a piecewise function we can include the physical measured data. In this case the measured variable contribution is extracted from the PWL file and
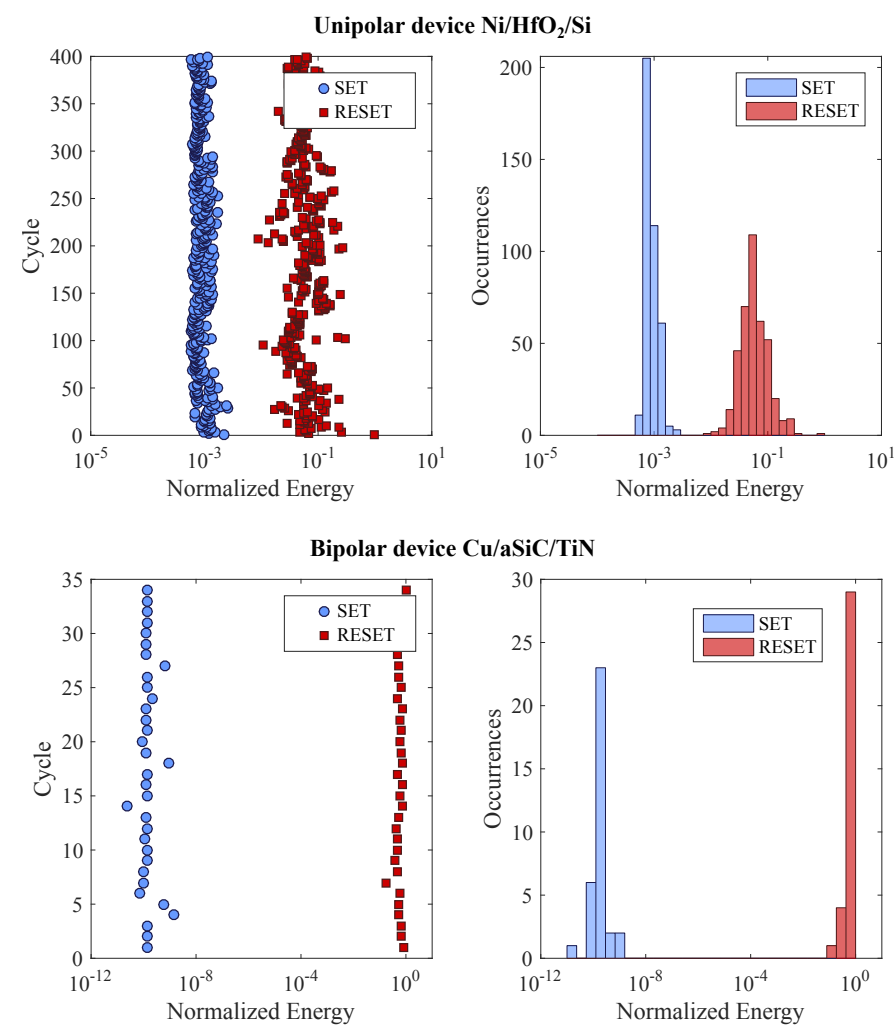

Fig. 8. SET and RESET scatter and histogram of measured energy levels required to perform switching in bipolar and unipolar devices. The values have been normalized using the maximum value of both processes.

injected using an additional source. An alternative to the PWL defined sources are the specific noisy source components that allow values defined via input files.

The above scheme covers two variability types: InterDevice Variability and Read Current Fluctuations variability. As an additional feature, the proposed modeling scheme admits Intra-Device Variability, defining how variability changes throughout time/cycling. This also provides the ability to define how the device gets degraded depending on its workload, and consequently, the determinability of its stored data. Therefore, by simulating consecutive switching events, the resistance state retention can be studied. Using the device state vector $s$ we can access at each moment the number of cycles and/or events experienced by the device, and provide a more accurate variability/degradation modeling:

1) Variability dependency on time or cycle number is extracted from physical measurements [36].

2) Variability is modeled using time/cycle dependent user defined functions. Therefore, the statistical characteristics such as standard deviation $(\sigma)$ and mean $(\mu)$ values become dynamic $(\sigma(t), \mu(t))$.

Finally, as reflected in equation 13 , the explicit declaration of the different conduction mechanisms eases the tuning of variability and degradation functions at each resistive level. The complex, level and time dependent variability characteristics shown in the results of Section IV-C take advantage of the proposed variability modeling scheme. 

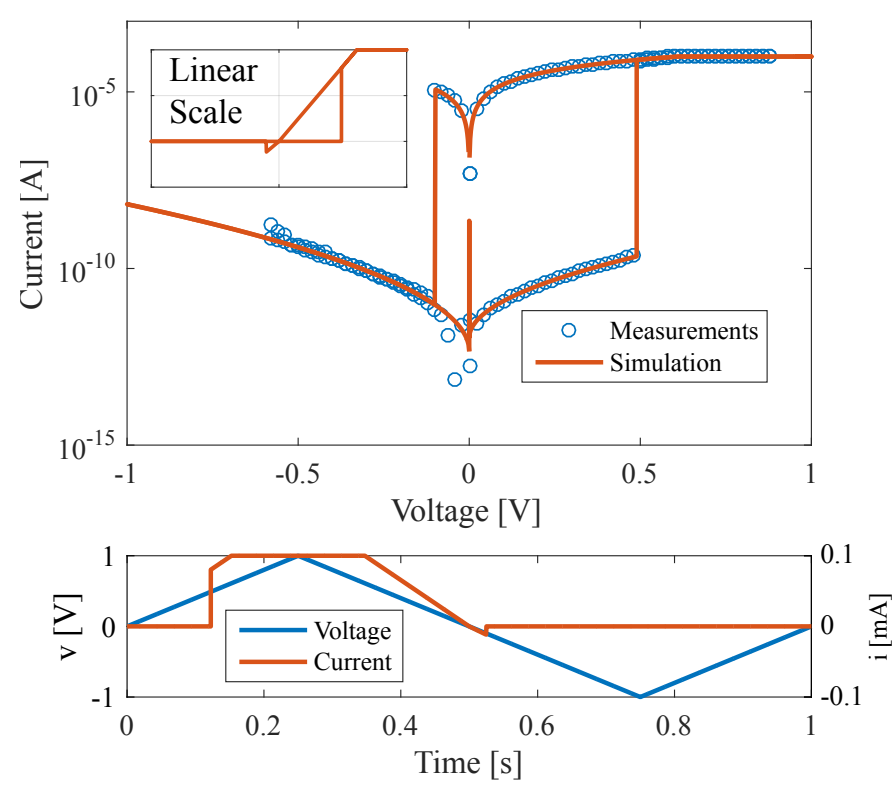

(a) Voltage-current relationship together with the voltage-current transients. On the upper graph the different slopes show how asymmetric behavior, depending on the input voltage polarity, was perfectly mimicked.

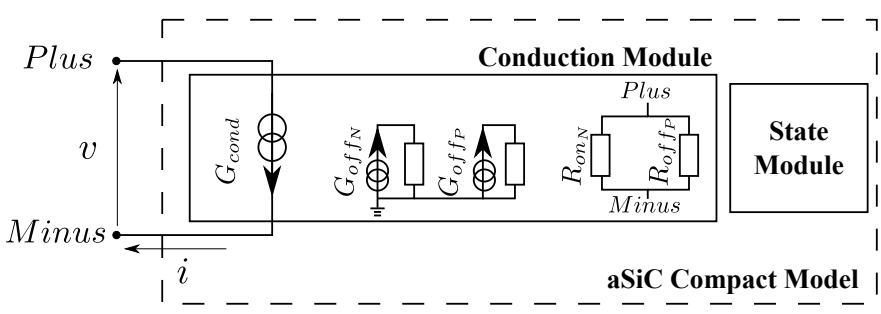

(b) Subcircuit using four different conduction submodules: off and on states for both positive and negative voltages.

Fig. 9. Bipolar device fitting: $v-i$ curves together with the used circuit.

\section{Physical Devices Simulations}

To show the model capabilities, two physical devices have been studied and fitted: a bipolar $\mathrm{a}-\mathrm{SiCCu}-\mathrm{TiN}$ and a unipolar $\mathrm{NiHfO}_{2} \mathrm{Si}$ device. Figure 8 shows the measured energy levels required to perform consecutive SET-RESET processes in both devices. The simulations shown in this section have been performed using Cadence Spectre circuit simulator, replicating the measurement experiments applied to the physical devices. The full source code of those models, together with their SPICE and Spectre implementations can be found in [25]. The parameter set fitting was automatically accomplished using $M A F$ simulator [1].

\section{A. Bipolar Device}

The first fitted device shows a reverse Schottky emission as its basic $H R S$ conduction process, even though it displays a leakage phenomena. The model is able to fit a minimum asymmetric behavior regarding its polarity. The $L R S$ is modeled using two different resistors depending on the voltage polarity. Figure 9 presents the simulated subcircuit and the achieved fitting compared against the measured data. The simulated voltage follows the stimulus that fed the device during its characterization.
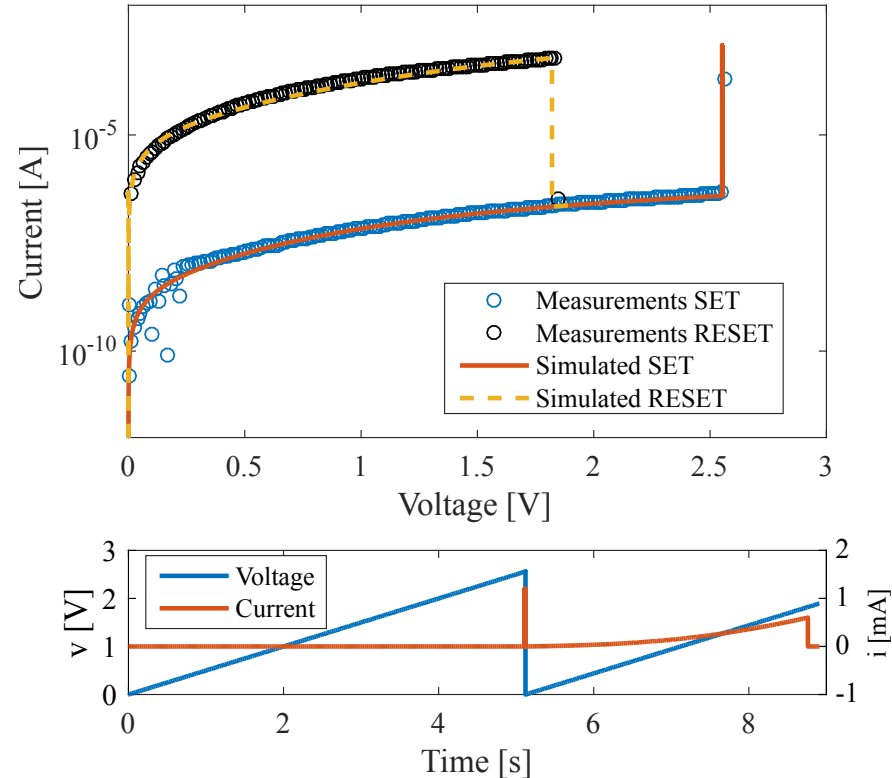

(a) Voltage-current relationship together with the voltage-current transients. The measurement method imposes the input voltage waveform: right after the SET is accomplished the sawtooth wave is reset to perform the next switch.

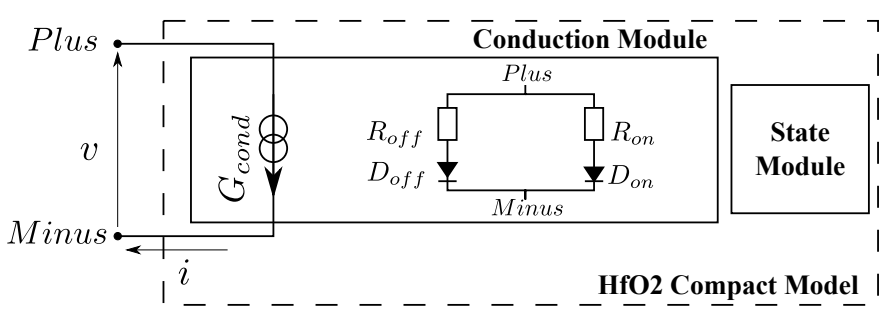

(b) Subcircuit using two resistor-diode submodules for off and on states.

Fig. 10. Unipolar device fitting: $v-i$ curves together with the used circuit.

\section{B. Unipolar Device}

The state handling approach described by system (8) perfectly fits the behavior of unipolar devices as shown in Figure 10a. The conductivity modeling follows the guidelines from [9]: two pairs of in series resistance-diodes match both $L R S$ and $H R S$ (Figure 10b). In this case the simulation input voltage reproduces the sawtooth stimulus feeding the memristor during the measurements.

\section{Variability}

The present section provides a simple example on how variability can be included within a specific device modeling. Two representative kinds of variability are considered: random telegraph noise and intra-device variability.

Using the random values $r_{\text {noise }}$ obtained from the normal distribution given by a characterized noisy voltage source, we shape the introduced RTN to affect in a higher grade the lower currents [34]:

$$
i= \begin{cases}i_{p}(v)\left(1+k_{0}\left(v_{0}-v\right)^{p_{0}} r_{n o i s e}\right) & \text { if } s^{(1)}=s_{p} \\ i_{o n}(v)\left(1+k_{1}\left(v_{1}-v\right)^{p_{1}} r_{n o i s e}\right) & \text { if } s^{(1)}=s_{\text {on }} \\ i_{\text {off }}(v)\left(1+k_{2}\left(v_{2}-v\right)^{p_{2}} r_{\text {noise }}\right) & \text { if } s^{(1)}=s_{\text {off }}\end{cases}
$$




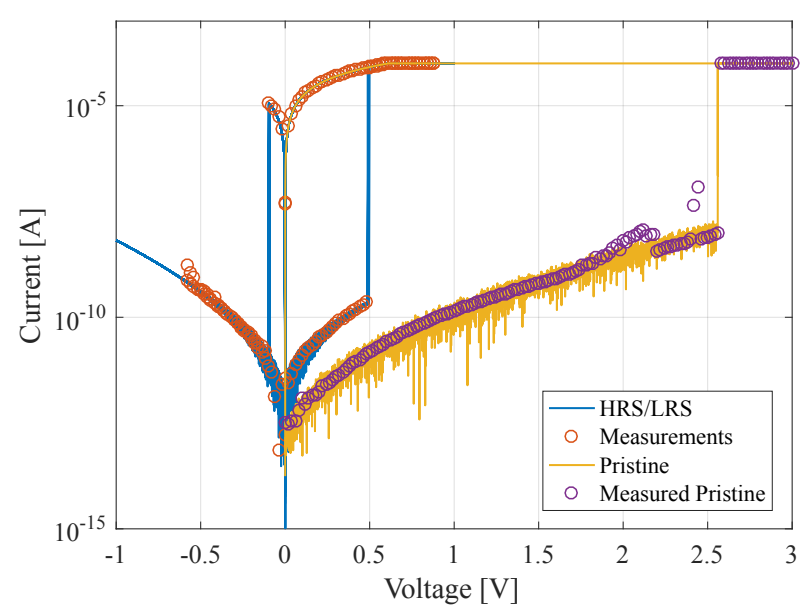

(a) Bipolar device suffering random telegraph noise over 50 cycles. The included pristine state is also affected by this variability phenomena.

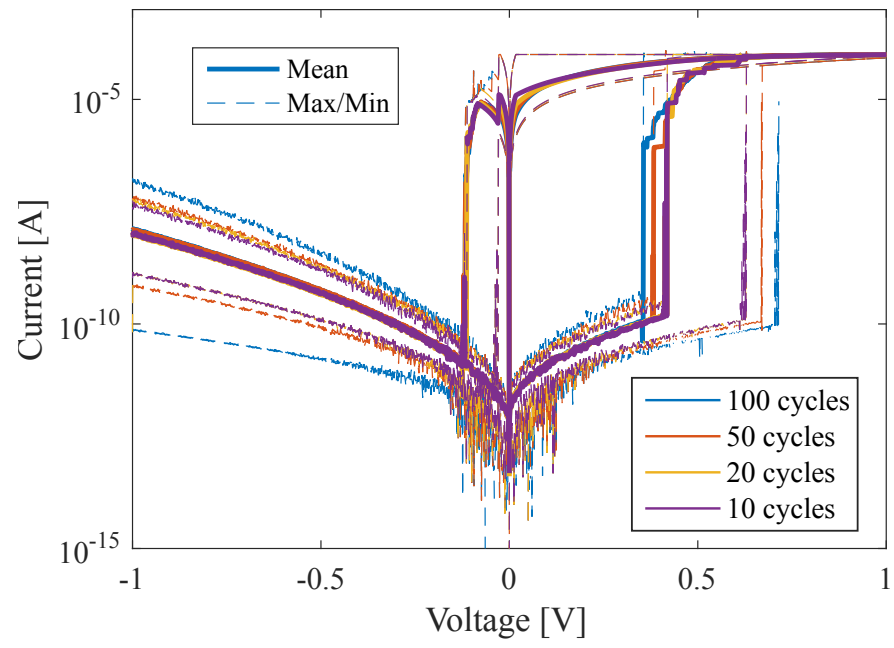

(b) Bipolar device with variability on its conduction mechanisms: intra-device variability (cycle/event dependent) and random telegraph noise. Mean and $\mathrm{max} / \mathrm{min}$ values are shown for 10 to 100 consecutive cycle experiments.

Fig. 11. Different examples of variability/degradation modeling.

Here the fitting parameters $k_{j}$ (with $k_{1}<k_{2}$ ), $v_{j}$ and $p_{j}$ allow the generation of the variability effects shown in Figure $11 \mathrm{a}$ for each pristine, $L R S$ and $H R S$ states.

The second modeled variability effect regards the conduction parameters on both the LRS and the HRS. In this case the parameters, cycle/time dependent in order to describe the device degradation, are extracted from PWL functions containing the normal distributed values and then the RTN is added. Figure $11 \mathrm{~b}$ presents the fingerprint of ten to one hundred consecutive cycles with these variability characteristics. The mean current-voltage relationship is represented together with the maximum and minimum values achieved during the experiment. It can be seen how the device current response varies with the cycling, widening the curves data and illustrating the device degradation.

\section{CONCLUSIONS}

We provided a customizable, physical memristor SPICE compact model, being able to accurately fit both unipolar

and bipolar devices without requiring a Verilog-A compatible simulator. The conduction modeling allows multi-level description, each level being able to be characterized using diverse contributions. The component state modeling is based on the process that trigger resistive switching (device electrical thresholds, energy, charge or flux), while omitting complex geometry/internal process computations.

This design simplifies the arduous work of translating physical device characteristics to the circuit compact model, and therefore helps device manufacturers to simulate state of the art devices. Not only is the model able to handle variability but it also describes how variability/parameter degradation evolves with time, making durability simulations straightforward. Its modular scheme allows phenomena such as temperature effects to be considered, improving memristor SPICE simulations and making the analysis more reliable. Finally, the model has been validated against two different physical devices.

\section{ACKNOWLEDGMENTS}

The authors would like to thank Mireia Bargallo and Francesca Campabadal from the IMB-CNM for providing the unipolar device samples, Montserrat Nafría and Javier Martín Martínez from the UAB for their support in the measurement of unipolar devices and valuable suggestions. We also want to acknowledge Kees De Groot, Katrina Morgan and Junqing Fan, from Southampton University, for supplying the bipolar devices, and also Ricardo Riaza from the UPM, for their helpful discussions.

\section{APPENDIX \\ Multilevel Device LTSPICE Code}

To illustrate the proposed model structure, we present the LTSP ICE source code related to the memristor characterized in Figure 2. Additional resources such as the full source code of charge controlled memristor, unipolar and bipolar devices, temperature dependency and variability aware experiments can be downloaded from http://vlsi.die.upm.es/memristor_spice_ model Compatible simulators: Linear Technologies LTSPICE and Cadence spectre.

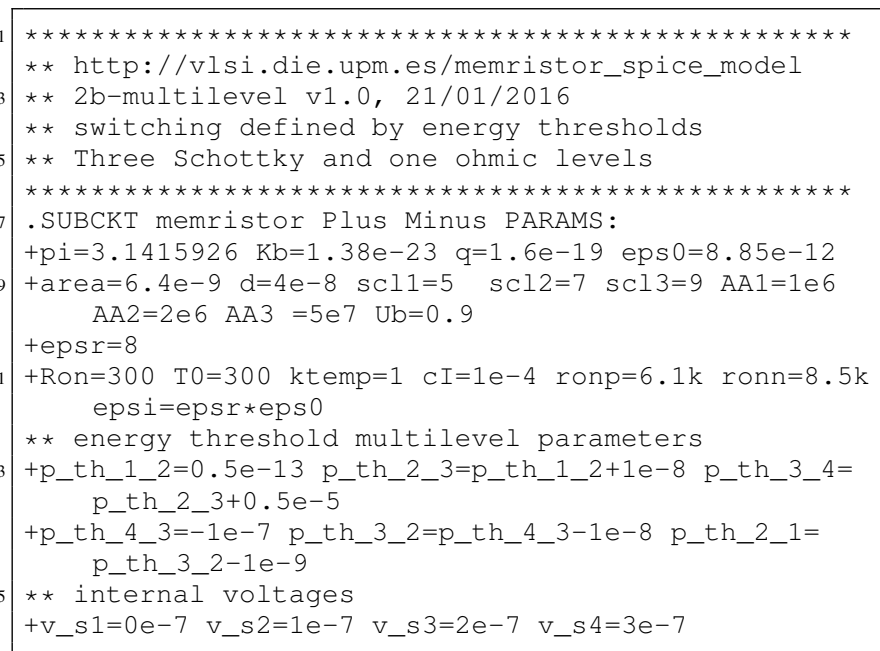




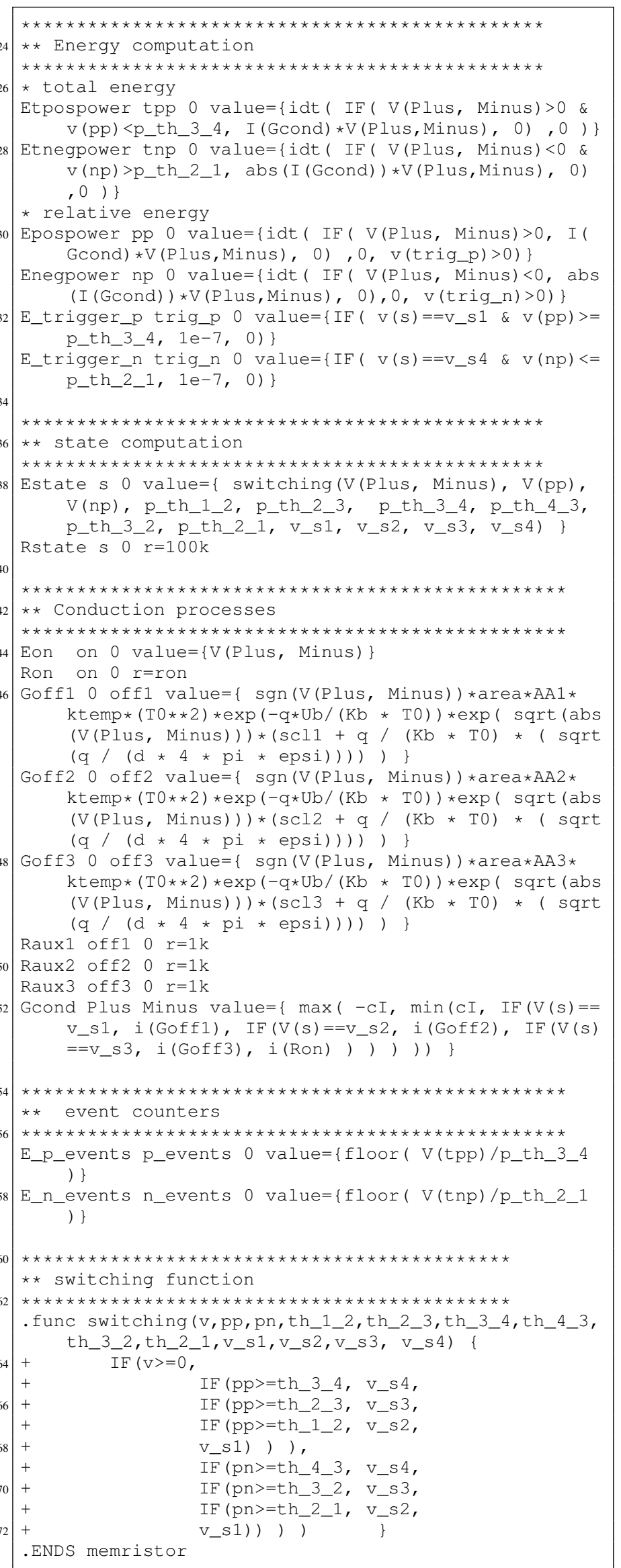

\section{REFERENCES}

[1] F. García-Redondo, M. Lopez-Vallejo, and P. Ituero, "Building Memristor Applications: From Device Model to Circuit Design," IEEE Trans. Nanotechnol., vol. 13, no. 6, pp. 1154-1162, nov 2014.

[2] J. Blasco, N. Ghenzi, J. Suñé, P. Levy, and E. Miranda, "Equivalent circuit modeling of the bistable conduction characteristics in electroformed thin dielectric films," Microelectron. Reliab., vol. 55, no. 1, pp. 1-14, jan 2015.

[3] Z. Biolek, D. Biolek, and V. Biolková, "SPICE Model of Memristor with Nonlinear Dopant Drift," Radioengineering, vol. 18, no. 2, pp. 210-214, 2009.

[4] K. D. Xu et al., "Two Memristor SPICE Models and Their Applications in Microwave Devices," IEEE Trans. Nanotechnol., vol. 13, no. 3, pp. 607-616, may 2014.

[5] J. Zha, H. Huang, and Y. Liu, "Novel Window Function for Memristor Model With Application in Programming Analog Circuits," IEEE Trans. Circuits Syst. II Express Briefs, vol. 7747, no. c, pp. 1-1, 2015.

[6] A. Ascoli et al., "The Art of Finding Accurate Memristor Model Solutions," IEEE J. Emerg. Sel. Top. Circuits Syst., vol. 5, no. 2, pp. 133-142, jun 2015.

[7] H. Li et al., "Variation-Aware, Reliability-Emphasized Design and Optimization of RRAM Using SPICE Model," in Des. Autom. Test Eur. Conf. Exhib. (DATE), 2015, 2015, pp. 1425-1430.

[8] J. F. Kang et al., "Modeling and design optimization of ReRAM," in 20th Asia South Pacific Des. Autom. Conf., vol. 1, 2015, pp. 576-581.

[9] E. Miranda, "Compact Model for the Major and Minor Hysteretic I-V Loops in Nonlinear Memristive Devices," IEEE Trans. Nanotechnol., vol. 14, no. 5, pp. 787-789, sep 2015.

[10] P.-y. Chen and S. Yu, "Compact Modeling of RRAM Devices and Its Applications in 1T1R and 1S1R Array Design," IEEE Trans. Electron Devices, vol. 62, no. 12, pp. 4022-4028, dec 2015.

[11] C. Yakopcic, T. M. Taha, G. Subramanyam, and R. E. Pino, "Generalized Memristive Device SPICE Model and its Application in Circuit Design," IEEE Trans. Comput. Des. Integr. Circuits Syst., vol. 32, no. 8, pp. 12011214, aug 2013.

[12] L. Zheng, S. Shin, and S.-m. S. Kang, "Modular Structure of Compact Model for Memristive Devices," IEEE Trans. Circuits Syst. I Regul. Pap., vol. 61, no. 5, pp. 1390-1399, may 2014.

[13] S. Kvatinsky, M. Ramadan, E. G. Friedman, and A. Kolodny, "VTEAM: A General Model for Voltage-Controlled Memristors," IEEE Trans. Circuits Syst. II Express Briefs, vol. 62, no. 8, pp. 786-790, aug 2015.

[14] C. Yakopcic, M. McLean, T. Taha, R. Hasan, and D. Palmer, "Memristorbased neuron circuit and method for applying learning algorithm in SPICE," Electron. Lett., vol. 50, no. 7, pp. 492-494, mar 2014.

[15] S. Kvatinsky et al., "TEAM : ThrEshold Adaptive Memristor Model," Circuits Syst. I Regul. Pap. IEEE Trans., vol. 60, no. 1, pp. 211-221, 2013.

[16] W. Chen et al., "Switching characteristics of W/Zr/HfO2/TiN ReRAM devices for multi-level cell non-volatile memory applications," Semicond. Sci. Technol., vol. 30, no. 7, p. 075002, 2015.

[17] P. Yan et al., "Conducting mechanisms of forming-free $\mathrm{TiW} / \mathrm{Cu} 2 \mathrm{O} / \mathrm{Cu}$ memristive devices," Appl. Phys. Lett., vol. 107, no. 8, p. 083501, aug 2015.

[18] L. Zhong, L. Jiang, R. Huang, and C. H. De Groot, "Nonpolar resistive switching in $\mathrm{Cu} / \mathrm{SiC} / \mathrm{Au}$ non-volatile resistive memory devices," Appl. Phys. Lett., vol. 104, no. 9, pp. 1-5, 2014.

[19] J. Blasco, N. Ghenzi, J. Suae, P. Levy, and E. Miranda, "Modeling of the hysteretic I-V characteristics of TiO2-based resistive switches using the generalized diode equation," IEEE Electron Device Lett., vol. 35, no. 3, pp. 390-392, 2014.

[20] H. Aziza et al., "Oxide based resistive RAM: ON/OFF resistance analysis versus circuit variability," in 2014 IEEE Int. Symp. Defect Fault Toler. VLSI Nanotechnol. Syst. IEEE, oct 2014, pp. 81-85.

[21] K. A. Morgan et al., "Switching kinetics of SiC resistive memory for harsh environments," AIP Adv., vol. 5, no. 7, p. 077121, jul 2015.

[22] Y. Liu et al., "Percolation mechanism through trapping/de-trapping process at defect states for resistive switching devices with structure of Ag/SixC1-x/p-Si," J. Appl. Phys., vol. 116, no. 6, p. 064505, 2014.

[23] L. Zhang et al., "Low voltage two-state-variable memristor model of vacancy-drift resistive switches," Appl. Phys. A, vol. 119, no. 1, pp. $1-9$, apr 2015.

[24] F. Kurnia, C. U. Jung, B. W. Lee, and C. Liu, "Compliance current induced non-reversible transition from unipolar to bipolar resistive switching in a $\mathrm{Cu} / \mathrm{TaOx} / \mathrm{Pt}$ structure," Appl. Phys. Lett., vol. 107, no. 7, p. 073501, aug 2015. 
[25] F. García-Redondo, M. López-Vallejo, and P. Royer, "LSI Online CAD Resources," 2015. [Online]. Available: http://vlsi.die.upm.es/resources

[26] M. P. Sah et al., "A Generic Model of Memristors With Parasitic Components," IEEE Trans. Circuits Syst. I Regul. Pap., vol. 62, no. 3, pp. 891-898, mar 2015.

[27] C.-m. Jung, K.-h. Jo, E.-s. Lee, H. M. Vo, and K.-s. Min, "ZeroSleep-Leakage Flip-Flop Circuit With Conditional-Storing Memristor Retention Latch," IEEE Trans. Nanotechnol., vol. 11, no. 2, pp. 360366, mar 2012.

[28] C. M. Jung, J. M. Choi, and K. S. Min, "Two-step write scheme for reducing sneak-path leakage in complementary memristor array," IEEE Trans. Nanotechnol., vol. 11, no. 3, pp. 611-618, 2012.

[29] C. Yakopcic, T. M. Taha, G. Subramanyam, and R. E. Pino, "Memristor SPICE model and crossbar simulation based on devices with nanosecond switching time," Proc. Int. Jt. Conf. Neural Networks, 2013.

[30] G. Ghosh and M. K. Orlowski, "Write and Erase Threshold Voltage Interdependence in Resistive Switching Memory Cells," IEEE Trans. Electron Devices, vol. 62, no. 9, pp. 2850-2856, sep 2015.

[31] M. Maestro et al., "Analysis of Set and Reset mechanisms in Ni/HfO2based RRAM with fast ramped voltages," Microelectron. Eng., vol. 147, pp. 176-179, nov 2015

[32] E. Yalon, A. Gavrilov, S. Cohen, and D. Ritter, "Validation and Extension of Local Temperature Evaluation of Conductive Filaments in RRAM Devices," IEEE Trans. Electron Devices, vol. 62, no. 11, pp. 3671-3677, 2015

[33] A. Chen, "Utilizing the Variability of Resistive Random Access Memory to Implement Reconfigurable Physical Unclonable Functions," IEEE Electron Device Lett., vol. 36, no. 2, pp. 138-140, feb 2015.

[34] A. H. Edwards et al., "Reconfigurable Memristive Device Technologies," Proc. IEEE, vol. 103, no. 7, pp. 1004-1033, 2015.

[35] D. Garbin et al., "Resistive memory variability: A simplified trapassisted tunneling model," Solid. State. Electron., sep 2015.

[36] A. Grossi et al., "Impact of Intercell and Intracell Variability on Forming and Switching Parameters in RRAM Arrays," IEEE Trans. Electron Devices, pp. 1-1, 2015.

[37] A. Prakash et al., "Multi-state resistance switching and variability analysis of HfOx based RRAM for ultra-high density memory applications," in 2015 Int. Symp. Next-Generation Electron., vol. 27. IEEE, may 2015, pp. 1-2.

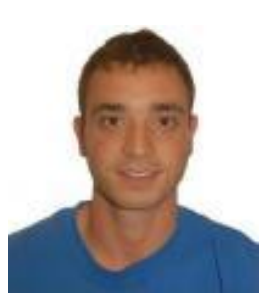

Albert Crespo Yepes, born in Barcelona (1982), he received the degree in Telecomunications Engineering in 2008 for UAB (Universitat Autònoma de Barcelona). In 2009 he obtained the master in micro and nano electronics. Recently (2012), he finalized his Ph. D. studies in Electronic Engineering in the group of Reliability Electron DEvices and Cricuits in the Electronic Department of the Universitat Autònoma de Barcelona (UAB). Currently, he is post-Ph.D. researcher in this group. His work is focused on the study of Dielectric Breakdown and Breakdown Reversibility characterization in MOS transistors with ultrathin high-k gate stack, Resistive Switching phenomena, Channel Hot-Carriers (CHC) and Bias Temperature Instability (BTI).

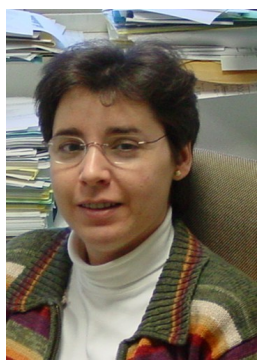

Marisa López-Vallejo (M'00) received the M.S. and Ph.D. degrees from the Universidad Politécnica de Madrid, Madrid, Spain, in 1993 and 1999, respectively. She is an Associate Professor with the Department of Electronic Engineering, Universidad Politécnica de Madrid. She was with the Lucent Technologies, Bell Laboratories, Murray Hill, NJ, as a Technical Staff Member. Her current research interests include low-power, process voltage, temperature-aware designs, computer-aided diagnostic methods and tools, and application-specific highperformance programmable architectures.

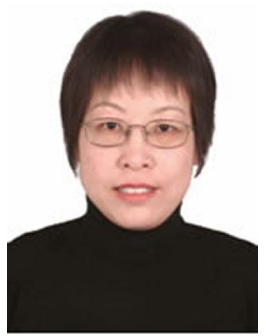

Liudi Jiang is Professor of Materials and Electromechanical Systems at the University of Southampton. She obtained BEng in Microelectronics, MSc in Physics. She received Ph.D. in advanced materials from the University of Dundee in 2002. She was appointed Lecturer in 2008 at the University of Southampton, Associate Professor in 2013 and then Professor in 2015. She is a Chartered Physicist. Current research interests include novel micro/nano-electromechanical systems (MEMS/NEMS), advanced resistive memories, biomedical sensor systems, functional materials and devices.

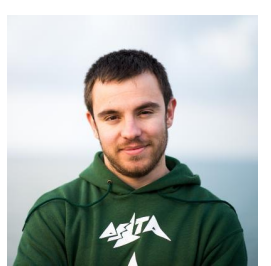

Fernando García-Redondo graduated from the Technical University of Madrid in 2011 with a degree in Telecommunication Engineering. Next, he focused on electronics obtaining a Master of Science in 2012. Currently he is a PhD candidate at the Integrated Systems Laboratory, UPM. His main research lines are RRAM modeling and simulation, reliable circuit design including PVT and radiation and novel circuit simulation approaches.

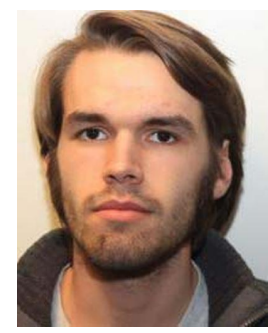

Robert P. Gowers received his MEng from the University of Cambridge in July 2014, with his focus being in Electrical Engineering. Areas of research Robert has previously been involved with include: flexible thin film transistors, the inkjet printing of liquid crystals, resistive memory. 